\title{
Sustitución de la harina de trigo por harina de quinua y puré de espinaca en la elaboración de una pasta enriquecida y fortificada.
}

\author{
Replacement of wheat flour by quinua flour and puré de spinaca in the elaboration of an enriched \\ and fortified pasta. \\ ${ }^{1}$ Ydania Espinoza Bardales ${ }^{\mathrm{a}},{ }^{1}$ Norma Elizabeth Gamarra Ramírez ${ }^{\mathrm{a}},{ }^{1}$ Rosario Esther Tarazona Minaya ${ }^{\mathrm{a}}$
}

\section{RESUMEN}

El presente trabajo de Investigación tiene como objeto de estudio la elaboración de la pasta enriquecida y fortificada. Este objeto de estudio tiene como problema el no conocer la elaboración de una pasta enriquecida y fortificada. Para lo cual se tiene como objetivo general elaborar una pasta enriquecida y fortificada sustituyendo la harina de trigo por harina de quinua y espinaca. Para la elaboración de la pasta alimenticia enriquecida se sustituyó la harina de trigo por harina de quinua caracterizada en proporciones de 10, 20 y $30 \%$. Seleccionándose pastas con mayor firmeza y menores perdidas de solidos solubles.

Finalmente se obtuvo una pasta enriquecida y fortificada con puré de espinaca, caracterizándose física y microbiológicamente. Concluyéndose que la harina de trigo y harina de quinua fue extra e integral respectivamente. El mejor tratamiento fue con sustitución de $10 \%$ de harina de trigo por harina de quinua, obteniéndose una pasta con una proteína, humedad y acidez dentro de las normas vigentes y rica en calcio.

Palabras clave: Sustitución, Harina, pasta.

\begin{abstract}
The present work of Investigation has like object of study the elaboration of the enriched and fortified pasta. This object of study has the problem of not knowing the preparation of an enriched and fortified pasta. For this purpose, the general objective is to make an enriched and fortified pasta by replacing the wheat flour with quinoa and spinach flour. For the elaboration of the enriched food paste, the wheat flour was substituted by quinoa flour characterized in proportions of 10, 20 and $30 \%$. Selecting pastes with greater firmness and lower losses of soluble solids.

Finally, an enriched pasta fortified with spinach puree was obtained, characterizing it physically and microbiologically. Concluding that the wheat flour and quinoa flour was extra and integral respectively. The best treatment was with substitution of $10 \%$ wheat flour for quinoa flour, obtaining a paste with a protein, humidity and acidity within the current standards and rich in calcium.
\end{abstract}

Keywords: Substitution, Flour, pasta.

${ }^{1}$ Universidad Nacional Santiago Antúnez de Mayolo, Huaraz - Perú.

${ }^{\mathrm{a}}$ Ingeniero de industrias alimentarias 


\section{INTRODUCCIÓN}

El trigo es una cariópside muy importante en la alimentación del ser humano. El Perú importa este cereal alrededor del 95\%; puesto que los trigos nacionales son de textura blanda, los cuales no aportan para elaborar pastas, galletas y pan de buena calidad., que son fuentes básicamente de carbohidratos y proteína deficiente en lisina.

Las pastas secas después del pan son el producto de demanda en nuestro medio y han sido motivo de investigación sobre su elaboración sustituyendo la harina de trigo por harinas sucedáneas como cereales (cebada, maíz, centeno, avena, arroz, quinua, kiwicha), leguminosas y tubérculos. En esta investigación se elabora la pasta a base de sustitución de la harina de trigo por harina de quinua. Entre los antecedentes del presente trabajo podemos citar a Rivera (2015) y Rojas (2013) que sustituyeron harina de trigo por harina de quinua en $(15 \%) \mathrm{y}$ $20 \%$ respectivamente, obteniéndose pastas de buena calidad.

La sustitución de la harina de trigo por quinua como fuente de lisina y de espinaca como fuente de calcio y hierro contribuye a elevar el valor nutricional de las pastas, al generarse mejoras en la cantidad y calidad de su proteína debido a una complementación de aminoácidos esenciales. Adicionalmente, la inclusión de quinua, contribuye a la recuperación y valoración de la quinua, disminuyendo así la dependencia del trigo.

Debido a este problema se plantea obtener nuevas pastas alimenticias enriquecidas $y$ fortificadas, utilizando la harina integral de quinua por su aporte en proteínas y fibra, así como la espinaca por su aporte en minerales como hierro y calcio para su consumo.
El trabajo presenta como objetivos específicos: Caracterizar las harinas de trigo y quinua, obtener la pasta enriquecida por sustitución de trigo por quinua, elaborar la pasta enriquecida y fortificada por adición de puré de espinaca y caracterizar el mejor tratamiento.

\section{MATERIALES Y MÉTODOS}

El tipo de investigación es experimental. La población está constituida por las harinas de trigo y quinua comercial, y espinaca fresca, producidos y obtenidos del ámbito del estudio. La formulación para la elaboración de la pasta enriquecida y fortificada se muestra en la tabla 1 y 2 respectivamente y el flujo de operaciones para la elaboración de pastas se muestra en la figura 1.

En la tabla 3, se presenta el diseño experimental de sustitución de la harina de trigo por harina de quinua y puré de espinaca en la elaboración de una pasta enriquecida y fortificada.

Tabla 1. Formulación para la elaboración de pasta enriquecida

\begin{tabular}{ccccc}
\hline Harinas & $\mathrm{M}_{0}$ & $\mathrm{M}_{1}$ & $\mathrm{M}_{2}$ & $\mathrm{M}_{3}$ \\
\hline Fase sólida (70\%) & & & & \\
Harina de trigo & 100 & 90 & 80 & 70 \\
Harina de quinua & 0 & 10 & 20 & 30 \\
Fase líquida (30\%) & & & & \\
Agua & 85 & 85 & 85 & 85 \\
Huevos & 15 & 15 & 15 & 15 \\
Puré espinaca & 00 & 00 & 00 & 00 \\
\hline
\end{tabular}

FUENTE: INTI(2008). 


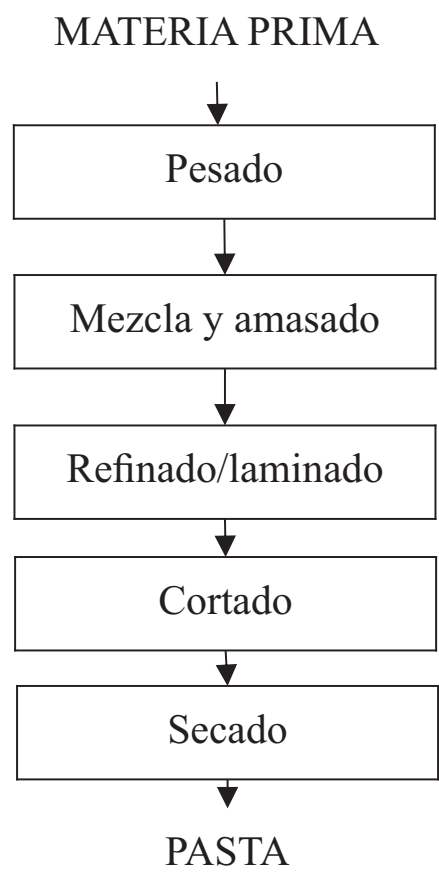

Figura 1:Flujo de Operaciones para la Elaboración de Pasta

FUENTE. Kent (1987)

Tabla 2. Formulación para la Elaboración de Pasta Alimenticia enriquecida y Fortificada

\begin{tabular}{ccccc}
\hline Harinas & $\mathrm{M}_{0}$ & $\mathrm{M}_{1}$ & $\mathrm{M}_{2}$ & $\mathrm{M}_{3}$ \\
\hline Fase sólida (70\%) & & & & \\
Harina de trigo & 100 & 90 & 80 & 70 \\
Harina de quinua & 0 & 10 & 20 & 30 \\
Fase líquida (30\%) & & & & \\
Agua & 85 & 70 & 70 & 70 \\
Huevos & 15 & 15 & 15 & 15 \\
Puré de espinaca & 0 & 15 & 15 & 15 \\
\hline
\end{tabular}

FUENTE: INTI (2008) 


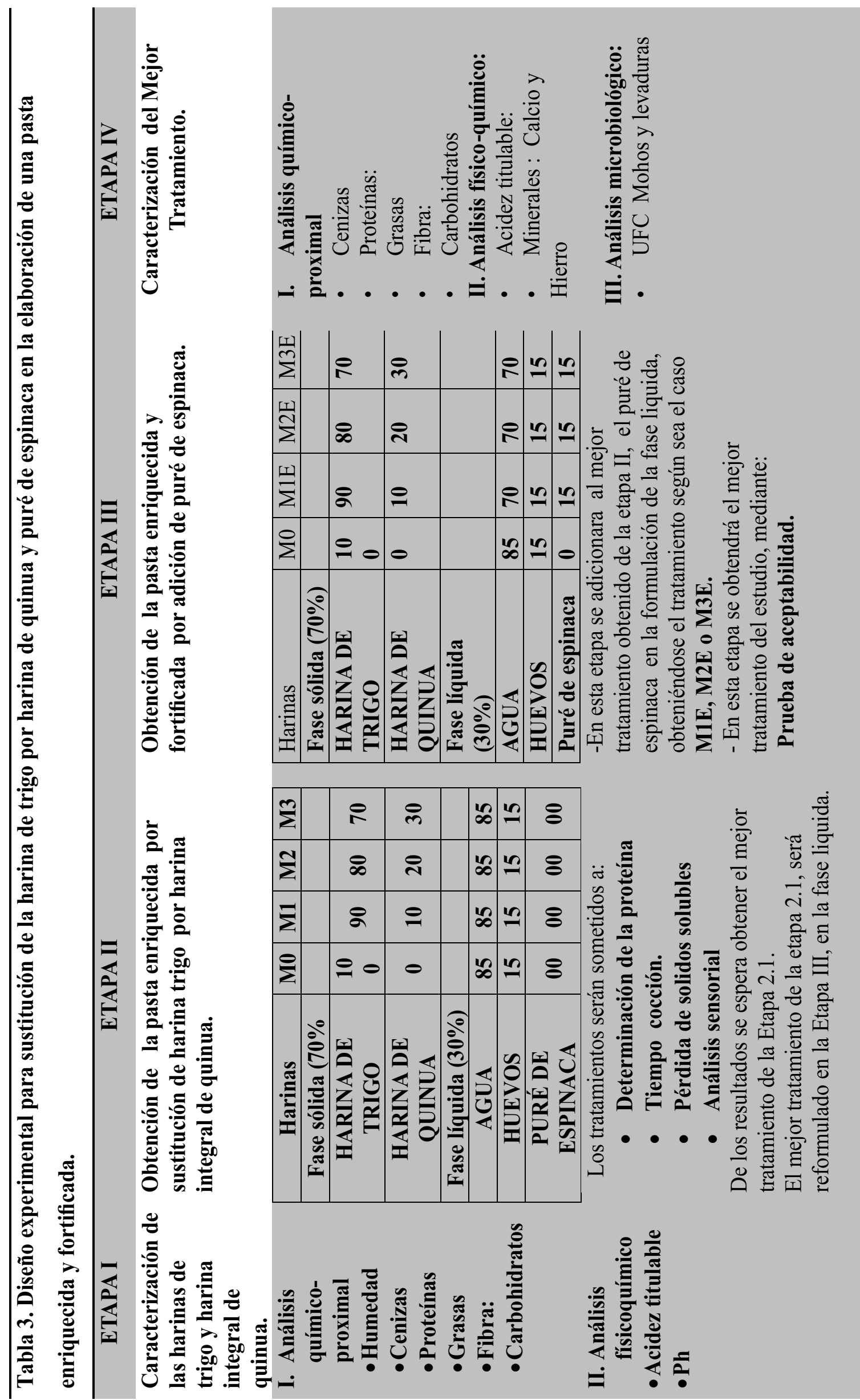




\section{RESULTADOS}

1. Caracterización de la harina de trigo extra y harina de quinua comercial.

En la tabla 4, se muestran los resultados.

Tabla 4. Análisis físico químico de la harina extra de trigo y harina de quinua

\begin{tabular}{|c|c|c|c|c|c|}
\hline $\begin{array}{c}\text { Componente } \\
(\%)\end{array}$ & $\begin{array}{c}\text { Harina } \\
\text { Trigo } \\
\text { Extra }\end{array}$ & $\begin{array}{c}\text { Harina Trigo } \\
\text { (Collazos, } 199 \\
\text { 3) }\end{array}$ & $\begin{array}{c}\text { Harina } \\
\text { Trigo } \\
\text { (Kent,198 } \\
7)\end{array}$ & $\begin{array}{c}\text { Harina } \\
\text { Quinua } \\
\text { comercial }\end{array}$ & $\begin{array}{c}\text { Harina } \\
\text { Quinua } \\
\text { (Collazos, 199 } \\
\text { 3) }\end{array}$ \\
\hline Humedad & 15 & 10.8 & $13-15$ & 10.49 & 13.10 \\
\hline Proteína & 12 & 10.5 & $8-13$ & 12.26 & 11.9 \\
\hline Grasa & 1.13 & 2.0 & $0.9-1.4$ & 4.47 & 4.7 \\
\hline Ceniza & 0.66 & 0.4 & $0.3-0.5$ & 2.42 & 2.9 \\
\hline Fibra & 0.15 & 1.5 & $0.1-0.3$ & 3.03 & 5.6 \\
\hline ELN & 71.06 & 74.8 & $65-70$ & 67.33 & 67.6 \\
\hline Acidez (a. s.) & 0.11 & - & - & 0.53 & - \\
\hline pH & 6.12 & - & - & 6.43 & - \\
\hline
\end{tabular}

2. Pasta enriquecida por sustitución de harina de trigo por harina de quinua.

En la tabla 5, 6 y 7 se muestran los resultados.

Tabla 5. Contenido de proteína

\begin{tabular}{ccccc}
\hline Componente & $\mathbf{M}_{\mathbf{0}}$ & $\mathbf{M}_{1}$ & $\mathbf{M}_{2}$ & $\mathbf{M}_{3}$ \\
\hline Proteína & 14.23 & 14.16 & 14.00 & 13.98 \\
\hline
\end{tabular}

Tabla 6. Tiempo de Cocción

\begin{tabular}{cccccc}
\hline Componente & $\begin{array}{c}\text { Muestra } \\
\text { Comercial }\end{array}$ & $\begin{array}{c}\mathbf{M}_{0} \\
\text { (Testigo) }\end{array}$ & $\mathbf{M}_{1}$ & $\mathbf{M}_{2}$ & $\mathbf{M}_{3}$ \\
\hline Tiempo (Min.) & 12 & 13 & 16 & 15 & 14 \\
\hline
\end{tabular}

TABLA 7. Pérdida de Sólidos solubles (S. S)

\begin{tabular}{cccccc}
\hline Componente & $\begin{array}{c}\text { Muestra } \\
\text { comercial }\end{array}$ & $\mathbf{M}_{\mathbf{0}}$ & $\mathbf{M}_{1}$ & $\mathbf{M}_{2}$ & $\mathbf{M}_{3}$ \\
\hline Pérdida de SS (\%) & 5 & 10.00 & 15.00 & 13.78 & 11.48 \\
\hline
\end{tabular}


3. Pasta enriquecida y fortificada por adición de puré de espinaca.

Con la formulación de la tabla 2 y figura 1, se elabora la pasta enriquecida y fortificada. En la tabla 8, se presenta la prueba de aceptabilidad de la muestra M3 y muestra comercial (Mc).

TABLA 8. Prueba de aceptabilidad.

\begin{tabular}{lcccccc}
\hline & color & Olor & Sabor & Textura & Apariencia & Aceptabilidad \\
\hline $\mathbf{M}_{3}$ & 4 & 2 & 2 & 3 & 3 & 3 \\
$\mathbf{M}_{\mathbf{c}}$ & 5 & 5 & 5 & 5 & 5 & 5 \\
\hline
\end{tabular}

\section{Caracterización del mejor tratamiento.}

La tabla 9y 10 muestran el análisis físico-químico y microbiológico.

TABLA 9: Análisis físico químico del mejor tratamiento.

\begin{tabular}{|c|c|c|c|c|}
\hline $\begin{array}{c}\text { Componente } \\
(\%)\end{array}$ & $\begin{array}{c}\text { Pasta enriquecida } \\
\text { y fortificada }\end{array}$ & $\begin{array}{c}\text { Pasta } \\
\text { Comercial } \\
\text { (Sahua,1995) }\end{array}$ & $\begin{array}{c}\text { Pasta } \\
\text { enriquecida } \\
\text { (Rivera,2012) }\end{array}$ & NTP \\
\hline Humedad & 13 & 12 & 12.5 & 15 \\
\hline Proteína & 13.0 & 12.59 & 13.2 & $11-13$ \\
\hline Grasa & 3 & 0.8 & 3 & $0.5-2$ \\
\hline Ceniza & 3 & 1.89 & 2.5 & - \\
\hline Fibra & 2 & 1.32 & 1 & 0 \\
\hline ELN & 65.5 & 71.4 & 75.5 & 71.76 \\
\hline Acidez (ac. lác) & 0.2 & - & 0.38 & 0.45 \\
\hline pH & 6.42 & - & 6.38 & - \\
\hline Calcio & $450 \mathrm{mg}$ & - & $98 \mathrm{mg}$ & - \\
\hline Hierro & $0.13 \mathrm{mg}$. & - & $1.95 \mathrm{mg}$ & - \\
\hline
\end{tabular}


TABLA 10. Análisis microbiológico del mejor tratamiento

Componente $\quad$ Pasta enriquecida y fortificada

Mohos y levaduras (UFC/g) Ausencia

\section{DISCUSIÓN}

Los resultados de la harina de quinua en comparación con la harina de trigo presenta mayor contenido en proteína, grasa, ceniza y fibra, coincidiendo con lo reportado por Collazos (1993).La composición química de la harina de trigo extra comercial se encuentra dentro de los rangos de la harina con $72 \%$ de extracción presentado por Kent (1987), mientras que la harina de quinua comercial, se encuentra cercano a los valores presentado por Collazos (1993).

La harina de trigo comercial es una harina extra, la cual se valida mediante los valores de acidez y ceniza (ITINTEC, 1975). Mientras que la harina de quinua comercial es una harina integral.

En los resultados se observa que a mayor sustitución de la harina de trigo por la harina de quinua va aumentando el contenido de proteína. Al respecto Según Espinoza citado por Tapia(1979), reporta que el contenido de la proteína se encuentra en mayor cantidad en el embrión de la semilla que en el perisperma. La diferencia está en la proporción del peso del embrión con el grano, que en la quinua es del $30 \%$, mientras que en el trigo corresponde al $1 \%$. Ello trae como consecuencia que el $70 \%$ del nitrógeno de la semilla de la quinua se encuentra en el embrión, mientras que en el trigo solo se encuentra del 2 al $4 \%$. Además es una harina integral, que su contenido de proteína es tan igual al del grano entero.
Repo - Carrasco (2000), reporta que la proteína de la quinua es mejor en cantidad y calidad que la proteína del trigo, refrendado también por Collazos (1993) por lo tanto la sustitución de la harina de trigo por harina de quinua permitirá que exista mayor contenido de proteína. Del mismo modo, Repo Carrasco (2000) reporta que la importancia de las proteínas de estas plantas andinas (Quinua, Kañiwa) radica en la calidad de las proteínas. Las proteínas de la quinua (45\%), como también las de kañiwa (41\%) son principalmente del tipo albumina y globulina. Estas, tienen una composición balanceada de aminoácidos esenciales parecida a la composición aminoacidica de la caseína, la proteína de la leche.

Respecto al tiempo de cocción de los diferentes tratamientos, se observa que a mayor sustitución corresponde un mayor tiempo de cocción. Coincidiendo con Rivera (2012), Rojas (2013), Cerrate (1989) y Sahua (1995).El tiempo de cocción está en función del tiempo de hidratación y gelificacion, requiriéndose más tiempo de cocción con muestras de mayor sustitución. El tiempo de cocción de la muestra comercial fue de12 minutos y 13 minutos para la muestra testigo. Mientras que para las sustituciones de harina de trigo por harina de quinua de $10 \%, 20 \%$ y $30 \%$ fue de 14,15 y 16 minutos respectivamente.

En lo referente al porcentaje de sólidos totales de los tratamientos, la muestra comercial presenta $5 \%$, frente a $10 \%$ de la muestra testigo. 
Las sustituciones de harina de trigo por harina de quinua de 10,20 y $30 \%$ presentan valores de $11.40,13.78$ y $15 \%$ de Sólidos totales respectivamente. Las pastas durante la cocción evidencian altas pérdidas de sólidos, acentuada pegajosidad y difícil desenrollamiento con sustituciones de 20 y $30 \%$; Por el contrario las pastas a menores niveles de sustitución (10\%) mantuvieron su firmeza, característica tanto por su enrollamiento como por su mínima pegajosidad entre las cintas.

Winston (1971) mencionado por Cerrate (1989), reporta pérdidas de $10.3 \%$ en espagueti con $100 \%$ de semolina, afirma además que ocurren perdidas de sólidos cuando las pastas son elaboradas con harina de alta extracción, puesto que la pérdida de sólidos indica la desintegración de la pasta durante la cocción. En el trabajo la harina de trigo es una harina extra fina pero la harina de quinua es una harina integral que ha influido en la pérdida de sólidos durante la cocción de la pasta; igualmente lo sustentan Rivera(2012)y Cerrate(1989).

En el caso del análisis sensorial se observa en el ANOVA para los tratamientos evaluados por los panelistas detectaron diferencias entre los tratamientos, por presentar un Fcal menor Ftab. En el TUKEY, para el primer atributo (color) los panelistas detectaron que los tratamientos M0, M1 y M2 son completamente diferentes, mientras que los tratamientos M0 y M3 son estadísticamente iguales. Para los atributos Textura, coloración y fractura los panelistas detectaron que todos los tratamientos son diferentes.

De los resultados evaluados el mejor tratamiento es M3 con $10 \%$ de sustitución de harina de trigo por harina integral de quinua, obteniéndose la pasta enriquecida. Para obtener la pasta enriquecida y fortificada al tratamiento
M3, se adiciono el puré de espinaca.

La prueba de aceptabilidad, se dio al tratamiento M3 y a la muestra comercial, resultando la muestra comercial con mejores valores en cuanto a los atributos color, olor, sabor, textura, apariencia y aceptabilidad.

La humedad de la pasta es menor al compararse a Sahua (1995), igual que Rivera (2012) por lo que, este se encuentra dentro de las exigencias de las Normas Técnicas.

La Proteína de la pasta se encuentra en los valores de Sahua(1995),Rivera(2012) y Normas respectivamente.

La grasa está por encima de los valores de la Norma, mayor que Sahua (1995) y cercano a Rivera (2012).El valor alto de la grasa es porque la harina comercial de quinua es una harina integral que tiene presencia de germen que influye en el valor final de la grasa de la pasta. Mientras que la fibra y la ceniza presentan valores altos comparados a Sahua (1995), Rivera (2012) y a la Norma Técnica, por ser una harina comercial integral de quinua.

La acidez, es un parámetro que está por debajo de Rivera (2012) y de la Norma Técnica.

En los resultados de los minerales, el calcio en la pasta sin espinaca es $98 \mathrm{mg}$ (Rivera ,2012) comparado al de la pasta que resulta ser mejor por contener a la espinaca y aporta un valor de $450 \mathrm{mg}$. Lo contrario sucede con el hierro, pasta sin espinaca aporta un valor de $1.95 \mathrm{mg}$. (Rivera ,2012), mientras que en la pasta con espinaca el valor es de $0.13 \mathrm{mg}$; sucede que la espinaca tiene presencia de ácido oxálico y la harina integral de quinua presencia de ácido fitico, estos ácidos forman sales de oxalatos y fitatos que no ayudan a la absorción de este mineral. 


\section{CONCLUSIONES}

La composición química tanto de la harina de trigo y quinua están dentro de los rangos presentado por Kent (1987) y Collazos (1993) respectivamente. La harina de trigo comercial es una harina extra y la harina de quinua comercial es una harina integral.

El tratamiento M3 es la pasta enriquecida por sustitución de la harina de trigo por quinua, con contenido bajo de proteína. Las características físicas de la pasta enriquecida son mayores en cuanto a tiempo de cocción y pérdidas de sólidos solubles.

El $10 \%$ de sustitución de harina de trigo por harina de quinua integral es el mejor tratamiento (M3), siendo esta la pasta enriquecida y fortificada.

Los resultados de los análisis físico químicos de la pasta enriquecida y fortificad cumplen las normas. Los valores de humedad, proteína y acidez. En cambio los valores de fibra, ceniza y grasa es mayor comparado a la Norma. En cuanto a minerales como el calcio tiene un valor alto de $450 \mathrm{mg}$ y un valor bajísimo en cuanto a hierro $0.13 \mathrm{mg}$.

\section{REFERENCIAS BIBLIOGRÁFICAS}

AOAC (Association of Official Analytical Chemists) .2005. Official Methods of Analysis of AOAC International. Washington: DC.

Berna, Amalia. 1995. Sustitución parcial de la Harina de trigo integral por harina de Frijol Nuña para la elaboración de fideos. Tesis para optar el título de Ingeniero en Industrias alimentarias. Universidad Nacional agraria La Molina. Lima- Perú.

Cerrate, Emily .1995. Efecto de Sustitución del trigo por tres variedades de cebada en la elaboración de fideos. Tesis para optar el título de Ingeniero en Industrias Alimentarias. Universidad Nacional Agraria la Molina. Lima-Perú

Collazos, Carlos .1993. La Composición de los Alimentos Peruanos. Lima: Instituto Nacional de Nutrición.

Desroier, Norman.1996 Elementos de Tecnología de Alimentos. México: Continental S.A.

Espinoza, Ydania .1996. Procesamiento de la quinua por el método de expansión. Huaraz-Perú.

INDDA (Instituto Nacional de Desarrollo Agroindustrial). 1987. Elaboración de fideos con sustitución parcial de harina de trigo por harina pre cocida de maíz. La Molina. Lima-Perú.

INTI (Instituto Nacional Tecnológico Industrial). 2008. Pastas Frescas: tallarines de sémola con huevo. 1a ed. Buenos Aires.

Hoseney, Russell. 1991. Principios de Ciencia Tecnología de los Cereales. España:Acribia S.A.

ITINTEC (Norma Técnica Peruana 206.010). 1981. Pastas y Fideos para consumo humano.

ITINTEC (Norma Técnica Peruana 205.037). 1975. Información Nutricional de los fideos.

ITINTEC (Norma técnica Peruana 205.027).1975. Harina de trigo para consumo domestico y uso industrial.

Kent, Norman.1987. Tecnología de los cereales. España: Acribia S.A. 
Lees, R. 1992. Análisis Moderno de los Alimentos. España: Acribia S.A.

Moreiras y col. 2013. Tabla de composición de alimentos. Sociedad Española de Nutrición. España: Pirámide. http://www.sennutricion.org/es/2013/05/ 14/tablas-de-composicin-de-alimentosmoreiras-et-al. $>$ [Consulta: 14-032018].

Primo, Eduardo.1979. Química Agrícola III. Alimentos. Madrid. Alhambra

Repo- Carrasco, Ritva. 2000. Introducción a la Ciencia y Tecnología de cereales y de granos andinos. Lima- Perú.

Rivera, Esau. 2012, Elaboración de pastas secas con sustitución parcial de harina de quinua. Tesis para optar el título de ingeniero en Industrias Alimentarias. Universidad Nacional Santiago Antúnez de Mayolo. Huaraz-Ancash.

Rojas, Wilber. 2013. Elaboración de fideos enriquecidos a partir de la sustitución parcial de la harina de trigo (T:durum) por harina de quinua ( Chenopodium quinoa wild). Tesis para optar el título de Ingeniero en Industrias alimentarias. Universidad Nacional José María A r g u e d a s . L i m a - P e r ú . http://repositorio.unajma.edu.pe/handle/ 123456789/199. [Consulta: 2-3-2018].

Sahua, Guadalupe. 1995. Elaboración de fideos con sustitución parcial de harina de trigo por harina pre cocida de pituca. Tesis para optar el título de Ingeniero en Industrias alimentarias. Universidad Nacional agraria La Molina. LimaPerú.

Serna, Sergio. 2009. Química, almacenamiento e industrialización de los cereales. España:AGT

Tapia, Mario.1979.La quinua y la kañiwa Lima-Perú.

\section{CORRESPONDENCIA}

Mag. Espinoza Bardales Ydania

ydaniaes@hotmail.com 